\title{
Franco Volpi, "Hermética versus Hermenéutica. Sobre el intento de Heinrich Rombach de sobrepasar el pensar de Heidegger. Y la carta con la respuesta de Rombach.»
}

\author{
Hermetics versus Hermeneutics. \\ About Heinrich Rombach's Commitment \\ to Surpass Heidegger's Thought. \\ And Rombach's Responding Letter
}

\author{
GRACIELA MARTA CHICHI \\ CONICET, Universidad Nacional de La Plata, Argentina
}

Recibido: 11/II/2014 Aceptado definitivamente: 31/III/2014

\section{RESUMEN}

El artículo que se presenta aquí traducido se editó en alemán en un volumen homenaje al filósofo Heinrich Rombach, quien se ha sabido unido a la «escuela de Freiburg » (en Briesgau) y determinado por la conexión entre Husserl y Heidegger, que, a pesar de las diferencias, es Fenomenología. Amigos, colegas y discípulos del profesor Rombach, por entonces profesor activo de la Universidad de Würzburg, dejaron en ese volumen huellas de haber oteado brechas y caminos muy distintos, en pos de alcanzar espacios abiertos, libres y diversos, propios de

(C) Contrastes. Revista Internacional de Filosofia, vol. XX No 2 (2015), pp. 347-372. ISSN: 1136-4076 Licenciatura de Filosofía, Universidad de Málaga, Facultad de Filosofía y Letras Campus de Teatinos, E-29071 Málaga (España) 
un filosofar que se sabe estructural, intermundano e intercultural. La participación de Franco Volpi, que se concentró en el Welt und Gegenwelt de Rombach, caminó a la par de quienes transitaron el primero de los tres movimientos, que emprendió la filosofía de Rombach y que se llama Hermética filosófica. Inspirado, pues, por la lectura del Welt und Gegenwelt, Volpi defiende que con el proyecto de la hermética el pensar de Rombach aventaja, de modo radical, la Zeitkritik de Heidegger de los fundamentos epocales, en cuyo caso el profesor italiano pone a consideración el aporte de la Hermética en la tradición de la filosofía alemana, que alcanza al fundador de la escuela hermeneútica. La carta de respuesta de Rombach, por su lado, desmiente el haber tenido que ver con la Zeitkritik heideggeriana, y lleva a primer plano lo fundamental del fenómeno hermético, esto es, la unidad inmediata e inseparable de ser humano y realidad, lo cual acontece como el respectivo caminar juntos o a la par, y sería algo con lo cual se topa quien lo pueda ver. Hermética sería entonces el arte de ver esa unidad inmediata.

PALABRAS CLAVE

ROMBACH-HERMÉTICA-HERMENEÚTICA-FENOMENOLOGÍA-CONCREATIVO.

\begin{abstract}
This is a translation of an article originally published in German in a special issue dedicated to philosopher Heinrich Rombach. Rombach identified himself as a member of the "Freiburg School" in Breisgau, and delimited by the connection between Husserl and Heidegger, which, despite their differences is Phenomenology. Friends, colleagues and disciples of Professor Rombach, who was back then an active Professor at the University of Würzburg, left traces in this volume of having glimpsed very different paths and gaps in the pursuit of reaching free, open and diverse spaces, characteristic of a structural, intermundane, and intercultural way of doing philosophy. Franco Volpi's contribution moved along others who focused on the Rombach 's Welt und Gegenwelt, following the first of three movements undertaken by Rombach`s philosophy, namely philosophical Hermetics. Inspired by Welt und Gegenwelt, Volpi claims that with the hermetic project, the thought of Rombach surpasses, in a radical way, the Heideggerian Zeitkritik, and so the Italian Professor examines the contribution of the Hermetics in the tradition of German philosophy, that includes the founder of the school of hermeneutics. The letter with Rombach`s reply to Volpi`s claim, on the other hand, denies Rombach `s involvement with the Zeikritik. Instead, Rombach emphasizes the centrality of the hermetic phenomenon, that is, the immediate and inseparable unity of human being and reality, which happens as they respectively move along together or at the same time, understood by Rombach as a special discover for who can grasp it. According to this, Hermetic is the art of seeing that immediate unity.
\end{abstract}

KEYWORDS

ROMBACH-HERMETICS-HERMENEUTICS-PHENOMENOLOGY-CONCREATIVE 


\title{
Hermética versus Hermeneútica. Sobre el intento de Heinrich Rombach de sobrepasar el pensar de Heidegger. Y la carta con la respuesta de Rombach.
}

\author{
FRANCO VOLPI ${ }^{1}$
}

[p. 251] Este TEXTO FUE ESCRITO EN 1983 inmediatamente después de la lectura de la Philosophische Hermetik de Rombach y apareció luego en el Philosophischer Literaturanzeiger (volumen 39 [1986] fascículo 2, 181-195). Rombach respondió con detalle a mis reflexiones en una carta del 20 de abril de 1986 que acá se publica. Esta contiene una sucinta presentación, que vuelve a poner en su sitio algunas cosas, deja entrever otras y, en conjunto, ofrece una concisa mirada general sobre el planteo de su pensar. La carta debería resultar interesante para todos los que siguen el pensar de Rombach. Mi texto -que luego apareció traducido también al húngaro en un fascículo especial de la revista de Budapest Athenaeum dedicado a la hermenéutica filosófica (volumen 1, [1992] Nr. 2, 223-241)- se publica aquí con algunas pocas actualizaciones en materia bibliográfica ahora junto con la carta de Rombach. De este modo, las dos voces del diálogo tienen la ocasión de hablar, lo cual puede llevar a que el lector se involucre de nuevo en otro diálogo, esta vez, con el asunto.

En todas partes hoy se considera anticuado el pensamiento absoluto en cualquiera de sus apariencias y máscaras. La crisis parece alcanzar no sólo el ideal de un saber de lo absoluto sino también la idea de una razón sustancial, e inclusive la idea de un saber estricto, irrebatible, universalmente válido,

1 Datos de la publicación alemana original: F. VOLPI, "Hermetik versus Hermeneutik. Zu Heinrich Rombachs Versuch, Heidegger weiterzudenken. Und Antwortbrief Rombachs", in: Georg STENGER, Margarete RÖHRIG (Herausgeber) Philosophie der Struktur-"Fahrzeug'" der Zukunft? Für Heinrich Rombach, Freiburg/München: Verlag Karl Alber 1995, pp. 251-276. Traducido al español por Graciela Marta CHICHI (CONICET, Universidad Nacional de La Plata, Argentina). Las notas a pie de la versión traducida quedaron corridas respecto de las notas originales de Volpi, a causa de haber añadido esta inicial (N. de la T.). 
que sea capaz de entender la realidad en su verdadero núcleo esencial. Ser y pensar están escindidos uno de otro en su completa heterogeneidad, y la complejidad del mundo escapa a todo entendimiento. En los propios bastiones de la teoría de la ciencia se deja caer el fundamentalismo a favor del falibilismo. Concepciones semánticas de la verdad resultan progresivamente acorraladas por teorías consensuales de la verdad. Se acepta casi como evidente la idea de que la verdad sea resultado de esfuerzos que se van acumulando, constructo del gran diálogo ininterrumpido de la humanidad, más precisamente, de la comunidad de investigadores, diálogo que ya no tiene por fin el acercamiento y la adecuación a lo real, un reflejo de la naturaleza, sino [p. 252] mera consistencia y coherencia de todos los posibles e innumerables juegos del lenguaje.

Aunque la propia representación cultural de la última etapa de la modernidad tenga todo esto por una adquisición de una forma más elevada y más aguda de conciencia, con todo, parece implicar una pérdida y una renuncia, una carencia, de la cual debe hacerse una virtud. No en último término se insinúa así un sentimiento de desazón, que va acompañando a la creciente autoconciencia de la modernidad, y que se expresa en la abundante literatura sobre la crisis y en las diversas críticas culturales de nuestro tiempo.

Cada vez más se consiente que la idea de razón, que se constituye programáticamente en origen de la modernidad europea, cuyo proyecto ésta desarrolla, es la causa de la pérdida de sentido que mientras tanto se experimenta casi de modo general, y la causa de la crisis de identidad de la civilización científico-tecnológica. En todas partes se construyen contra-culturas contra el mundo dominado por la economía, por la burocracia y por los trámites legales propios de la racionalización industrial y postindustrial; ellas le señalan sus límites e intentan restituir a éste integridad a través de la fundación de bases compactas de sentido contra las desoladas deformaciones de los espacios del mundo de la vida. Ante el agotamiento de la antigua razón sustancialista, ante su instrumentalización y formalización, el mandamiento nuevo se llama búsqueda de sentido.

La desazón del nihilismo y el síndrome de abstinencia aparejados a la atrofia de sentido tientan entonces, a menudo fácilmente, a adoptar de modo abrupto un orden y una afirmación decisionista de imperativos parciales de valor y de sentido, llevan al anhelo romántico de lo originario o se proyectan utópicamente en el anuncio salvador de sentido. Panaceas se ofrecen a montones y a gusto. Pero la modernidad, que ha comido del árbol del conocimiento, apenas puede llegar a saciarse con esto. El gusano de la sospecha consume de modo irrefrenable cualquier hallazgo sustancial de sentido. El contenido transparente de la verdad de lo dicho resulta enturbiado y eclipsado por la conciencia atenta y altamente sensible de lo no-dicho que penetra en ella. La semántica cultural de filosofía y ciencia, de moral y política, de mito, arte y religión junto con sus 
grandes ideales de verdad, de [p. 253] bien y mal y de Dios es socavada por la presunción, que se abre paso furtivamente, de que ella no dice exactamente lo que piensa sino que remite a un fundamento más profundo, por el cual las verdades de la superficie cobran el sentido propio que las funda, el cual reside, sin embargo, en la falta de claridad de lo no-dicho, y que, como tal, ya siempre se sustrae a poder ser completamente interpretado. La conciencia crítica de la modernidad se extiende así entre un escepticismo autoinmune, taimado al modo racionalista y agrandado por la escuela de la sospecha, y una necesidad de sentido cultivada mediante abstinencia, ascetismo y vacío nihilista.

Heidegger es quien, de modo magistralmente lúcido, ha entendido, cuestionado e intentado restablecerse del estado patológico en que se encuentra la modernidad. El nivel de radicalidad y de vuelta al fundamento, que él ha alcanzado en su pensar y en su diagnóstico del presente, es tan alto que parece sumamente difícil tomar posesión de su herencia o poder ir más lejos que él. En la mayoría de los intentos asumidos hasta aquí pueden constatarse más recaídas que progresos, aplanamientos en vez de perfiles, planteos morigerados y atenuados en lugar de aquellos que profundicen y radicalicen la cuestión. Quien asuma pensar más allá de Heidegger ya hace mucho, si logra mantenerse en su nivel.

Entre las pocas excepciones, en las cuales el intento de seguir pensando más allá de Heidegger parece significativo y fructífero, se cuenta sin lugar a dudas el pensar de Heinrich Rombach. La vasta extensión que considera su proyecto filosófico y el ímpetu de su apuesta destacan las investigaciones aquí alcanzadas en el sentido de la vuelta al fundamento y de la radicalidad del estilo heideggeriano de pensamiento. Al menos recordemos aquí las más importantes.

Ya el primer libro de Rombach: Die Gegenwart der Philosophie. Eine geschichtsphilosophische und philosophiegeschichtliche Studie über den Stand des philosophischen Fragens (Freiburg/ München 1962; 3. grundlegend neuarbeitete Auflage 1988) deja fácilmente reconocer de qué modo fuerte y radical él hace suyo el interés de Heidegger por ontología e historia, que al mismo tiempo busca también continuar pero sobre base propia. ${ }^{i}$ En efecto, Rombach comparte, por un lado, con Heidegger la convicción de que al pensamiento filosófico pertenecen la posibilidad y la tarea de interpretar de modo radical y fundamental [p. 254] el acontecer completo de la realidad, que solo puede ofrecer el saber de las ciencias particulares desde perspectivas parciales y regionales exclusivamente. Por otro lado, ya con ese primer arranque especulativo, que quiere aportar a la comprensión de sí y a la determinación de la ubicación del filosofar actual, Rombach intenta superar el filosofema de Heidegger, esto es, la orientación predominante de la idea de Heidegger de la historia del ser en los textos de los grandes filósofos, dado que aquél -Rombach- extiende su captura a otras experiencias fundamentales de la humanidad. 
El ejercicio espiritual del preguntar filosófico radical no entra en pleno juego allí donde éste vuelve a relacionarse consigo mismo, con su propia historia y representación de sí, sino allí donde penetra de modo profundo en todas las otras regiones del ser -religión, mito, arte, ciencia, moral, política-. ${ }^{2}$ En el marco de ese pensamiento, en su primer libro Rombach esclarece la experiencia fundamental del filosofar, y señala qué sitio e importancia relativa tiene en cada caso el pensar filosófico en la antigüedad, en el medioevo, en la época moderna y en el presente, cuándo se convierte en experiencia fundamental y cuándo, por otra parte, aparece como experiencia accesoria junto a otras experiencias fundamentales. De este modo ya se perfila el pensamiento dominante de la historia fundamental, que será determinante para el desarrollo del pensamiento posterior de Rombach: una concepción e interpretación de la historia, que quiere ser ontológicamente radical y que no aspira a ser mera reconstrucción, sino reconstitución, que no debe ser solo historia del ser ni debe apoyarse en textos filosóficos únicamente, como tampoco ella debe ser solamente historia del espíritu o -en oposición a esto- tampoco mera historia social, historia económica, historia política del poder. Antes bien, ella tiene que dejar expuesta la estructura fundamental, que sustenta el conjunto del acontecer y el plexo total de una época en todas sus manifestaciones; por ende, la estructura fundamental, en la cual está en juego en cierto modo el todo de una época, que sustenta todos sus aspectos parciales. En este sentido ella se llama historia fundamental, y soporta y se presupone en todas las historias particulares.

Con el intento de reconstruir con amplitud la historia fundamental europea se relaciona la investigación, que Rombach sienta en una trilogía, a saber: [p. 255] Substanz, System, Struktur. Die Ontologie des Funktionalismus und der philosophische Hintergrund der modernen Wissenschaft (2 Bde., Freiburg/

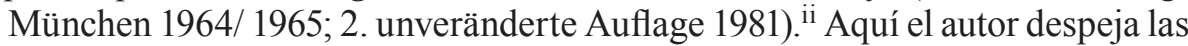
tres formas fundamentales de la vida que son características de Occidente. La forma fundamental de la sustancia impregna la antigüedad y el medioevo. Ella se muestra en la representación básica, según la cual el conjunto del cosmos, todo ser, cada acontecer resulta caracterizado mediante la diferencia básica entre un núcleo esencial estable e inmutable y la manifestación de una imagen mudable y perecedera, entre la presencia persistente de la forma y el perpetuo llegar a ser del acontecer, entre permanencia y temporalidad.

Sistema es el rasgo fundamental que caracteriza a la época moderna. Es el rasgo fundamental, que primero se vislumbra en la astronomía en el marco del giro copernicano, pero que pronto se convierte en rasgo fundamental de

2 Sobre este punto véase el primer trabajo de Rombach, Über Ursprung und Wesen der Frage, 2. Unveränderte Auflage mit einem Nachwort zur Neuausgabe, Freiburg/ München 1988 (1. Auflage 1952). 
la ciencia moderna por antonomasia, y que, finalmente, atraviesa e impregna la realidad en su conjunto. La representación dominante que subyace es que todo está estructurado en un plexo total de conexiones regulares y de procesos determinados, y que, en correspondencia con esto, aquello se deja captar científica y sistemáticamente y exponer de modo descriptivo. Si la ciencia es pensar en sistemas, entonces técnica es el actuar, que se orienta a pensar en sistemas y que viene guiado por éste. Ciencia y técnica, como pensar en sistemas, que se corresponde con actuar conforme a sistemas, impregnan tan fuerte la época moderna con el carácter de sistema que todo resulta, en último término, aprendido, interpretado y representado desde el inicio de modo sistemático. Inclusive el hombre y la convivencia humana son determinados sistemáticamente. Pero tan pronto como sistemas entran en conflicto, sin que las oposiciones del sistema dejen reducirse a otro sistema de un nivel superior que las resuelva, se desgarra y agota la realidad del sistema de la ciencia y de la técnica. Esto sucede, sobre todo, por el hecho de que el punto de vista de un sistema permanece atrapado en su propia limitación y, como tal, ofrece resistencia a cualquier intento de sacarlo de perspectiva, esto es, de llevarlo a un plano universal y totalizador. En este sentido, la forma fundamental del sistema es -según Rombach- ontológicamente inconsistente.

Esta inconsistencia pone en marcha un cambio, que lleva a otra forma fundamental de la vida: la estructura. Estructura es [p.256] la experiencia fundamental del presente y del futuro inmediato. Atendiendo a la historia del concepto, la categoría de estructura aparece ya a comienzos del medioevo y resulta agudamente caracterizada por Nicolás de Cusa; pero recién cuando la modernidad se complete, ella se convertirá en la experiencia fundamental que caracterice a la realidad en su conjunto. Justamente por eso, estructura llega a ser el tema principal para Rombach y la palabra fundamental de su pensamiento, el cual se entiende y se denomina ontología estructural o fenomenología estructural-ontológica. Con la cristalización de la categoría de estructura y con el cumplimiento del programa de una ontología estructural se relaciona el escrito principal de Rombach: Strukturontologie. Eine Phänomenologie der Freiheit (Freiburg/ München 1971; 2.unveränderte Auflage 1988).

En la palabra fundamental de estructura se concentran las experiencias de la ontología y de la historia: estructura es de hecho un modo de ser, una forma fundamental del ser, pero que abarca no solo la actualidad puntual de su presencia, sino también su nacimiento y muerte, por consiguiente, su cambio. Con esto intenta ponerse en correspondencia el modo de ver de la ontología estructural, mientras ella se declara genética, orgánica y concreativa ${ }^{\text {iii }}$, esto es: genética, porque ella no queda detenida en su presencia en ser rígido alguno, sino que capta de una vez la salida o la procedencia del ser; orgánica, porque ella busca superar la diferencia ontológica de parte y todo, que caracteriza el 
pensar al modo sistemático y busca así dar con la identidad ontológica de ambos o idemidad ${ }^{i v}$; concreativa es ella, por fin, porque con ello intenta recoger en un nivel superior de consideración la separación tradicional de hombre y naturaleza, en tanto ella enseña cómo ambos forman esencialmente un conjunto -desde el punto de vista de la ontología estructural y del de la historia fundamental-

Si este libro marca la irrupción de la ontología estructural, entonces la siguiente investigación Phänomenologie des gegenwärtigen Bewusstseins (Freiburg/München 1980) desea ofrecer comprensión de sí y aclarar la relación de la ontología estructural con la filosofía contemporánea. Sin entrar en las reflexiones que allí se desarrollan, su resultado puede resumirse declarando que la ontología estructural se confiesa como pensar en la tradición del pensamiento fenomenológico, que primaria y principalmente consiste en esclarecer y sacar a la luz la típica propia y la dinámica propia de los fenómenos. Pero esa confesión se estructura al mismo tiempo [p. 257] como intento de una comprensión de sí, que en la historia del movimiento fenomenológico se reconoce y pronuncia como incremento, es decir: en la fenomenología filosófico-trascendental de Husserl primero recibe aclaración fundamental la dimensión de la razón y la del conocimiento; a través de la fenomenología ontológico-existencial de Heidegger resultarían conceptualizados no sólo resultados homogéneos de la razón sino también la heterogeneidad de la razón, la facticidad y la historicidad de la vida humana; la fenomenología ontológico-estructural de Rombach, finalmente, desea llevar no solo al hombre en todas sus expresiones de vida y creaciones culturales sino a la realidad completa a un esclarecimiento radical de sí misma; con ello se potencia al mismo tiempo la idea de fenomenología con un pensamiento de procedencia hegeliana, según el cual la fenomenología se entiende a sí misma como el proceso de auto-esclarecimiento de los fenómenos, como el proceso a través del cual los fenómenos llegan a encontrar completa transparencia.

Si las investigaciones mencionadas hasta aquí se orientan principalmente al género tradicional de la prosa filosófica, en el libro ilustrado, que se titula Leben des Geistes. Ein Buch der Bilder zur Fundamentalgeschichte der Menschheit (Freiburg i.Br. 1977), Rombach inaugura y ensaya una nueva estrategia de exposición, con la cual debería ir mejor al encuentro del punto de partida de la historia fundamental. Con la estrategia consistente en analizar íconos culturales (Bildanalyse), Rombach captura el contenido de mitos, sagas, obras de arte, textos literarios y demás documentos culturales, con el fin de bosquejar experiencias fundamentales y estructuras, que marcan en cada caso las etapas de la historia fundamental.

Según la misma estrategia metodológica Rombach presenta ahora el proyecto de una hermética filosófica, que se anuncia algo llamativamente como "cambiar el pensamiento sobre la realidad" (umdenken über die Wirklichkeit) 
en el escrito Welt und Gegenwelt. Umdenken über die Wirklichkeit: die philosophische Hermetik (Basel 1983). ${ }^{\vee}$ Tan pretencioso como pueda sonar siempre esto, el hecho es que se trata de una empresa filosófica, que no deja nada que desear en lo que a radicalidad concierne. Puede considerárselo como un intento de aventajar, de modo radical, la crítica heideggeriana de los fundamentos epocales, pero justamente en el punto en el que el pensar de Heidegger se detiene cautelosamente ante la cuestión de una terapia eficaz para el estado patológico de la modernidad y así aquél parece quedar estancado. Si Heidegger [p. 258] anuncia que solo un dios puede salvarnos, y si por lo tanto adopta la actitud de abstinencia, que es el pensar rememorante (andenkende), y mantiene distancia de prescribir receta alguna, así la misma convicción de Rombach de que solo un dios pueda salvarnos ofrece la ocasión de buscar un nuevo dios y (la ocasión) de prestar atención a su mensaje. Si Heidegger es el pensador de un tiempo empobrecido, en el que los dioses huyeron y en el que aún no llega a atisbarse nueva divinidad, entonces Rombach quiere preparar la epifanía de ese nuevo dios o estar a punto de iniciarla. Si detrás de la heroica postura del pensar de Heidegger deja reconocerse el temple fundamental de un pesimismo radical, entonces Rombach alberga in pectore el optimismo más esperanzado. En correspondencia con esto se determina también el programa de la hermética filosófica: ella quiere mostrar las raíces de la alienación de la modernidad, despertar una conciencia "crítica" e "ilustrada" sobre la patología que de allí resulta y, finalmente, espera señalar el camino para revertir ese estado de enfermedad.

Por eso la hermética filosófica busca una forma de existencia perdida e intacta, a la cual uno podría aferrarse en la marcha actual de la crisis. Esta forma de existencia debe divisarse inmediatamente en la época homérica en la figura del dios Hermes. Rombach desea devolver actualidad a esa figura, a fin de que el desencantado mundo del retraimiento nihilista de sentido pueda conseguir una orientación. Hermética, entonces, también como presentación de una alternativa a la modernidad europea, cuyo paradigma no tiene que ser ahora inventado, sino la historia europea como posibilidad que se desplaza, pero que puede ser percibida a cada momento como acompañante permanente, y así pueda reconocerse ella en el recuerdo de la figura del dios Hermes y recobrar nueva vigencia.

Pero ¿por qué recurrir a la figura de un dios? La argumentación de Rombach no solo hace plausible ese recurso, sino también lo torna incitante: "La existencia humana es siempre solo posible sobre la base de un fundamento de vida, en el cual las más importantes tesis fundamentales sobre naturaleza e historia, verdad y valor, origen y fin se unen en un plexo armónico, en una doctrina primordial de vida y de existencia, que contenga las presuposiciones más importantes de una vida humana digna. Estas tesis fundamentales, modos de ver, [p. 259] hábitos de percepción y enseñanzas de vida forman un plexo, 
que mantiene vigencia en consonancia, y esto genera una buena concordancia del hombre con su mundo vital: una vida plena de sentido con un alto temple de ánimo. Esta estructura basal aparece al hombre antiguo no como teoría, no en forma de concepto, sino en la figura clara de un dios." (22) ${ }^{\mathrm{vi}}$ La argumentación prosigue luego: "pero ¿por qué justamente de un dios? Porque el hombre no pudo urdir a partir de sí mismo la figura fundamental de su propia vida, sino que debió toparse con ella en un hallazgo afortunado. La forma de vida, que se abre en una experiencia fundamental concordante, no se corresponde con ninguna estructura de necesidades dadas con anterioridad; antes bien, una vez dadas allí las posibilidades de satisfacción, ella recién abre una nueva estructura de necesidades, una existencia elevada -justamente una vida humana digna-. La forma fundamental no se encuentra en el mismo ser humano, sino en el Todo de la realidad, en el espíritu de un paisaje, en las fuerzas ascendentes del plexo total. En lo divino, por lo tanto. En consecuencia, el Dios." (p. 22).3vii

La marcha del pensamiento de Rombach gana más fuerza a través del hecho de que la vuelta a la figura del dios Hermes no ofrece ningún romanticismo arcaizante, ningún pensamiento que rememore lo que ha sido, o evocación nostálgica alguna de la unidad de la fuente originaria, sino que plantea tomar la posibilidad, que se deja identificar históricamente, pues, en el mito griego de Hermes, cuyo significado no reside igualmente en su facticidad histórica ni tampoco solo en su posición de valor en la historia fundamental, sino antes bien en el hecho de que ella representa una alternativa potencial a ser reconocida, que acompaña siempre a la historia occidental, sobre todo, cuando ella llegue a su culminación y a su completo acabamiento.

Percibir a Hermes como figura-símbolo de la forma de existencia alternativa que ha de asumirse, aunque hoy permanezca olvidada y oculta, exige -según Rombach- una ruptura con aquella elucidación, que simula valerse de la razón, cuya doctrina de la sospecha socava o mina cada donación de sentido; exige su correspondiente superación y la irrupción hacia una forma auténticamente más alta de ilustración, que haga justicia al conjunto de la realidad, no solo a las partes de la realidad que fueran homogéneas con la razón y [p. 260] que participen de la razón. Rombach aclara: "Tras el largo tiempo de la noche de los dioses y del fracaso de todo sistema de la razón, con los cuales debía instalarse la paz perpetua, pero que solo ha originado guerras cada vez más inhumanas, podría constituirse ahora la disposición de buscar una comunidad más viva, más creativa y diferenciada entre los pueblos, la cual fuese, sin embargo, más que un compromiso, a saber, un auténtico poner en juego las verdades fundamentales. No es necesario representar esas verdades fundamentales como dioses, sino

3 Respecto de la antropología filosófica de Rombach véase Strukturantropologie. "Der menschliche Mensch”, Freiburg/ München 1987. 
que es necesario ver en ellos algo importante e irreemplazable. La ligereza de la poca fe, propia de una ilustración de corto aliento, no nos puede tocar más. Lo que debe tener vigencia y duración debe venir de lejos y ser respetado en todo. Ser humano no es nada nimio. Es la cumbre de la vida y conserva su altura, solo cuando se hace cada vez más evidente su precio. Seguro que la conciencia de la altura y del valor no tiene necesariamente la figura del culto, pero esa conciencia debe conservarse y legarse en un ropaje claro, si es que el ser humano no debe hundirse en la barbarie, en una vida fantasmal, en la mera técnica del bienestar. En consecuencia, es necesario asegurarse de nuevo la altura original de las experiencias fundamentales de la vida, para que de ellas surja una comprensión superior de la existencia, que se aclare y ordene de un nuevo modo." (25)

Ahora bien, hermética debe significar también una ruptura con el conflicto interminable de visiones del mundo y de religiones, y de hecho en el sentido de llevar adelante y decidir la disputa occidental de los dioses: "Asi también es necesario", escribe Rombach, "que las religiones del mundo se encuentren en una nueva relación pacífica. Para ello hace falta más que extrema tolerancia, para ello hace falta una relación positiva entre las partes. Recién cuando las religiones terminen de disputarse mutuamente sus dioses, ganará la humanidad una nueva relación con lo divino. La cuestión es, en cambio, cómo dios es en cada caso el único y, no obstante, abierto a otros. También los dioses deben -así parece- aprender primero a hablar entre sí. Quizá sea Hermes el único adecuado para ello, el maestro divino. La hermética como teoría de la comprensión de lo no-concebible es el intento de reconciliar, de un nuevo modo, dioses, mundos y hombres entre si."(25)

[p.261] La fuerza de la pretensión de verdad que levanta es manifiesta. ¿Acaso también resulta cumplida esa pretensión? La misma pregunta ya se ha planteado en el caso del último Heidegger. Es decir, para muchos, en el lenguaje de Heidegger se unen una sobrecargada fuerza ilocucionaria, una pretensión de verdad, que se juega de modo marcado con un vacío de contenido proposicional. ¿Qué sucede en el caso de Rombach? A esa pregunta puede contestar de un modo significativo solo aquél, que esté dispuesto a entrar en el pensar de Rombach, que es digno de seguirse, cuando se lo lleva adelante en atenta discusión y comprensión del asunto. Por eso es difícil anticipar aquí una respuesta, pero afortunadamente también resulta innecesaria. Permítaseme solo una breve consideración: bien parece que el estar dispuesto a una orientación positiva de parte del lector se torna difícil, por el hecho de que la hermética filosófica, debido a su radical pretensión de verdad, no admite ninguna captatio benevolentiae, ningún compromiso ni mediación, y así puede llevar fácilmente a que se rechace su tono en parte mesiánico, profético. Ahora bien, quizá esto sea reparado a través del método de la filosofía que se sirve de ilustraciones, el 
cual abre sin duda un acceso fácil y provechoso a la médula filosófica de la hermética. Es decir, mientras que Rombach plantea una ruptura con la preferencia tradicional por los escritos de los filósofos y se abre en principio a cualquier semántica cultural, en tanto el autor amplía entonces la idea heideggeriana de la historia del ser a la exposición de la historia fundamental, puede dejar que en su hilo de pensamiento entren en acción las posibilidades del esclarecimiento plástico y la fuerza de convencimiento que tienen las imágenes de la interpretación de mitos, símbolos, obras de arte, documentos culturales y formas fundamentales de vida -no sin el peligro cierto de sobreinterpretarlos o de equivocarse en la interpretación-, tanto más fácilmente logrará predisponer (al lector) al mensaje filosófico de la hermética.

El mito de Hermes se sondea en todos sus contenidos y aspectos como reservorio de determinaciones, a fin de extraer de él la forma de vida y de comprensión, de la cual pueda gestarse el sentido salvador. Esto se encuentra en el primer planteo de la investigación (II, 21-52), que sigue a la exposición introductoria del programa de la hermética (I, 11-20). Los títulos de los parágrafos particulares de esa parte bastan para aludir a la complejidad de la figura, que se interpreta en sus bases y en su historia: Hermes se considera como [p. 262] dios de pastores, como dios del robo y del engaño, como dios del hallazgo, como dios con el báculo de serpiente, como dios de lo cifrado y como señor del tránsito, como dios del límite, como dios de las idiosincracias, como dios con miembro fálico erecto, como dios del vuelo, como el dios acompañado por Dionisos y Pan, como el tres veces grande (Hermes Trismegistos). Rombach sabe capturar todos esos aspectos en una imagen de conjunto, concordante y completa acerca del dios, la cual se convierte en paradigma elocuente de la forma originaria de la vida intacta y dichosa que se busca.

Sin embargo, contra esa forma de existencia originaria e intacta, que representa visualmente Hermes, se ha impuesto en la historia europea otra forma de vida opuesta. Ella se encarna en la imagen del hermano de Hermes, Apolo. El cambio radical se describe así: "Con el ascenso de Europa gobierna el principio apolíneo, de modo tal que ningún otro principio más fuese posible junto a él. El mito de Hermes y el culto de Hermes cayeron en el olvido, la cultura se acomodó a lo general, a lo abierto, a lo público, al orden y al dominio. Bajo el signo de ese principio, Europa tomó posesión del dominio cultural del mundo, esa cultura se instala, pues, sobre el principio de dominación. Con el ascenso de ciencia y técnica, que representan las formas más externas de la disposición de claridad apolínea y de la aspiración de orden, la forma europea del espíritu pareció haberse corrido completamente del círculo de cualquier posibilidad de ponerla en cuestión. Preguntar, esto debió darse de ahora en más solo en la forma de la ciencia; actuar tuvo por fin sentido solo en la figura de la técnica." (55) 
Rombach reconstruye las formas fundamentales y las etapas de la historia fundamental del destierro y del ocultamiento de Hermes (III, 53-81) como también -en reflejo con ello- el advenimiento y la afirmación de lo apolíneo en las formas de un ascenso progresivo del conocer, de la ciencia, de la técnica y del bienestar (IV, 83-105). Pero con el despliegue de lo apolíneo hasta su punto de culminación también se hace evidente y resulta efectiva la contradicción fundamental, que reside en él desde el comienzo, la cual deja expresarse con palabras de Rombach brevemente y sin rodeos así: "El mundo apolíneo es aquel mito, que ha venido a reemplazar todos los mitos por medio de Ilustración. Con ello se pierde el hecho de que la ilustración misma es todavía un mito." (p. 101). ${ }^{\text {viii }}$ Esta contradicción fundamental latente [p. 263] provoca luego en el mundo apolíneo numerosas contradicciones, que dejan reconocer también en la superficie del acontecer la caducidad fundamental de esa construcción del mundo y de la forma de existencia, que se corresponde con ella.

Rombach elige medicina, técnica, seguridad social, politica como ámbitos paradigmáticos, en los cuales esas contradicciones, que echan raíces en la contradicción fundamental de lo apolíneo, se hacen patente en formas más marcadas y agudas. "He aquí, por ejemplo, la medicina, que investiga los fenómenos de enfermedad y de salud, y que tantos conocimientos nos ha dado, por los cuales con derecho se la llega a tener en cuenta. De hecho, parece como si muchas enfermedades, bajo las cuales la humanidad antes tuvo mucho que padecer, hubiesen sido hoy derrotadas o estuvieran controladas y, mediante ello, la condición hubiese mejorado decididamente. Sin embargo, es así que, por eso, otras enfermedades han aparecido, daños de la civilización, por los cuales los humanos no sufren menos ni mueren menos frecuentemente que antes (...) Si se planteara la pregunta teniendo en cuenta dos o más generaciones, respecto de cuál de ellas, tomada en conjunto, fuese la más sana, siempre se tendría en cuenta la más antigua. Nuestros abuelos tenían ciertamente menos medicamentos, pero ellos estaban, a pesar de ello o justamente por eso, más sanos. Asimismo, su modo de estar enfermo era más sano. Lo contrario vale para las generaciones posteriores. Ellas están en conjunto más enfermas e inclusive su modo de estar sanos alberga enfermedad. Así vale que la medicina nos ha hecho en particular más sanos pero en general más enfermos. Contra esta enfermedad fundamental la medicina no tiene recurso, parece mucho más que todos sus medios trabajan en pro de la enfermedad fundamental." (101 y siguientes). "El mismo fenómeno contradictorio observamos bajo la perspectiva de la técnica. Si la técnica ha logrado que el ser humano esté libre de dificultades, también al mismo tiempo ella lo ha llenado de cargas más pesadas en conjunto. El hombre moderno no se siente más libre sino más atado, no más aliviado sino más lleno de cargas, no se ha librado del estrés sino inmerso en él de un modo total, que no se deja definir más sobre hechos particulares. $L a$ 
técnica nos ha liberado de carga en particular pero en conjunto y en grado más alto nos ha llenado de cargas. Esta es la contradicción." (102 y siguiente). "De nuevo el mismo fenómeno en el ámbito de la seguridad social. También acá en el mundo apolíneo se ha hecho mucho en particular - pero entonces con ello se ha destruido aun más en conjunto-." (103) "También en la política se enseña la contradicción [p. 264] fundamental. Todos quieren la paz, pero el conjunto se hizo más agresivo." (103). ${ }^{4}$

Rombach reduce a la contradicción fundamental del mundo apolíneo las contradicciones, que así crecen y proliferan en la modernidad europea, cuya conciencia crítica desgarran, provocan infelicidad y atomizan. Ya Hegel y Marx, Nietzsche y Heidegger han sido sismógrafos sensibles de las rupturas y trastornos de la modernidad, y la crítica de la época, que ellos practicaron, ha trazado también las dos posibles posiciones ejemplares básicas, que puede tomar esa crítica: del modo inclusivo, cuando absorbe y resiste dialécticamente, o del modo excluyente, cuando aleja y rechaza antidialécticamente. La crítica epocal (Zeitkritik) de Romach quiere ambas cosas: ella quiere lo uno sin dejar lo otro; porque contradicciones semejantes y principalmente la contradicción, que está a la base de éstas, no se dejan solucionar ni cortar de un golpe como a un nudo gordiano. La acomodación dialéctica mediadora asciende hasta el estado de cosas existente, y sólo agrava la contradicción fundamental: los contra-mundos, que aparecen hoy por todas partes en contra de la civilización técnico-científica de modo antidialéctico y excluyente, intentan por cierto cortar el nudo gordiano, pero ellos mismos se enredan finalmente en él. En el mejor de los casos, con su rebelión se deja expresar el malestar de la modernidad, pero sin poder conocer sus causas profundas y sin poder, por ello, siquiera rozar la campana de acero de lo apolíneo que circunda el mundo contemporáneo.

Dado que la hermética de Rombach no quiere plantear adaptación alguna a las relaciones existentes, tampoco quiere ser apología de esa clase de contra-mundos. Ella desea, en cambio, acumular las experiencias que están a la base de todos esos contra-mundos, para atribuirlos luego a la experiencia fundamental, a saber: la experiencia fundamental hermética, en la cual las estructuras profundas de la realidad, las leyes espontáneas de su acontecer resultan transparentes. Mediante esta experiencia fundamental llegan a hacerse transparentes las verdaderas causas más profundas de la distorsión apolínea como también el arraigamiento de esa distorsión en el acontecer fundamental hermético de la realidad.

4 Al respecto véase el artículo de Rombach "Philosophische Zeitkritik heute. Der gegenwärtige Umbruch im Lichte der Fundamentalgeschichte", en: Philosophisches Jahrbuch 92 (1985) 1-16. Además ahora: Phänomenologie des sozialen Lebens. Grundzüge einer Phänomenologischen Soziologie, Freiburg/ München 1994. 
[p. 265] La parte final de la investigación (V, 107-169) está dedicada a apresar y a delinear los contornos de la experiencia hermética fundamental. Se trata de una experiencia particular, que se sustrae al rapto del conocimiento y por ende tampoco se deja captar en trazos redondeados y sistemáticos. Ni dialéctica ni discurso pueden hacerle justicia. La descripción en prosa de los miles de caminos de la realidad no alcanza a dar con ella. Ella invade e irrumpe, se ilumina y apacigua en el temple lírico del surgimiento/emergencia (Ursprung) $^{\mathrm{ix}}$ encontrado/a. Solo señas, indicaciones, vistazos e inspecciones profundas pueden hacer que nos abramos a su ocurrencia contagiosa. La hermética filosófica quiere esto ante todo, y por eso detesta principalmente el querer demostrar paralizante, el deber de dar cuentas, la fatigosa tarea sin fin del argumentar; para establecer correspondencia con la experiencia hermética fundamental, la hermética debe ser un pensar contra la corriente, cuya agudeza solo se deja encontrar y gozar en una coejecución dispuesta.

Allí donde escurren las posibilidades de las costumbres del lenguaje filosófico de la tradición, se muestra apropiado el "método" de la filosofía de la imagen (Bildphilosophie), para abrir al nuevo nivel de sentido más básico y al mismo tiempo para facilitar el acompañamiento y el entendimiento de esa apertura. La variedad de interpretaciones ofrecidas, que deberían explorar la experiencia fundamental hermética, da una impresión de la amplitud del espectro de fenómenos capturados tanto como de la diversidad de las posibilidades de acceso abiertas con ello en la hermética filosófica. De modo hermético se interpretan, esto es, la hermética reclama para sí, sobre todo, figuras de la mitología griega (junto a Hermes, también Pan, Dionisos, Apolo, Ganimedes, entre otros más); piezas de la Biblia como la historia de David y Goliat o la de José y sus hermanos, la parábola del buen pastor o el significado de las figuras de ángeles; textos literarios de Hölderlin (Regreso al hogar), de Göthe (FaustoMefisto), de Schiller, de Thomas Mann (La montaña mágica); también mucho de la historia del arte es objeto de interpretación: por ejemplo, el grabado en madera de Durero El rezo de los Reyes Magos, El jardín de las delicias de Jerónimo Bosch, Monje junto al mar de Caspar David Friedrich, Nadador ciego y A la primera palabra límpida de Max Ernst, Techos rojos y El tiempo es un río sin orillas de Marc Chagall; también se interpretan documentos y fenómenos culturales [p.266] de toda clase, en cuyo caso es digno de notar que -debido al hecho de que la historia fundamental intenta superar el eurocentrismo de la filosofía tradicional- en igual medida también entren en consideración culturas orientales (véase, por ejemplo, la interpretación del camino del té de los japoneses o el significado de los golpes en el Budismo-Zen). ${ }^{5}$

5 Sobre el diálogo, que Rombach inició y fomentó, entre la cultura occidental y la oriental (al que no es posible entrar en esta ocasión), véase el volumen publicado en colaboración entre 
El lector debe forzosamente acudir él mismo al texto de Rombach y tomar conocimiento de esa y de las otras interpretaciones particulares, en las cuales se subraya en cada caso un aspecto fundamental de la experiencia hermética, y de ese modo tendrá también ocasión de seguir, de un modo más concreto y más vívido, la hermética en la marcha de la interpretación. Pero para cerrar cabe al menos determinar la posición de la hermética en conjunto tanto con respecto a su valor en el desarrollo del pensar de Rombach, como con respecto a su sitio en la marcha contemporánea de la filosofía.

En el contexto del desarrollo del pensar de Rombach, la hermética filosófica tiene un valor significativo; ella subraya de modo palmario otra nueva etapa de desarrollo y cierto punto culminante, desde el cual se echa una luz más nítida a la marcha hasta ese punto. Seguramente desde el comienzo se tuvo en la mira este akmé especulativo, sobre el cual se proyecta una elucidación básica sobre las estructuras profundas de la realidad, y Rombach ya había apuntado antes en esa dirección. Sin embargo, la realización del proyecto en la forma de una hermética filosófica resulta algo nuevo también para el lector, que ha seguido desde el comienzo el pensamiento de Rombach. A partir del avance de este nuevo punto de partida, es digno de recomendar al lector que renueve su lectura de los escritos de Rombach; para el propio Rombach esto exige agudizar la propia comprensión de sí.

Justamente, la rigurosa comprensión de sí, que logra Rombach a través de sentar y de probar las altas pretensiones de la hermética filosófica, al mismo tiempo lo lleva a determinar su lugar en la filosofía actual y a polemizar con planteos rivales. Al principio se recordó el hecho de que [p.267] en una detallada autocomprensión filosófico-histórica, a saber, en Phänomenologie des gegenwärtigen Bewusstseins, Rombach se confiesa partidario de la tradición de la fenomenología y, desde allí, intenta llevar más lejos el punto de partida fenomenológico de Husserl y, sobre todo, el de Heidegger a través de una radicalización ontólogico-estructural. Ese intento, cuyo pensamiento dominante transforma la idea de la historia del ser en la idea de la historia fundamental, y cuya intención es superar el filosofismo tradicional, el antropocentrismo y el eurocentrismo, que Husserl y Heidegger asumen sin haberlos puesto en duda, ese intento se tiende entre los dos polos de problemas, en los que se concentró Heidegger: de ontología e historia, de ser y tiempo, y con ello Rombach entra a competir sobre todo con otro intento, que hoy mucho se estima y se reconoce, al asumir la herencia de Heidegger en el horizonte de problemas de historia y ontología, a saber, con la hermenéutica filosófica.

En aguas de Heidegger la hermenéutica filosófica ha abierto y hecho útiles

él y K. Tsujimura y R. Ohashi: Sein und Nichts. Gundbilder westlichen und östlichen Denkens, Basel 1981. 
para el siglo las adquisiciones, en las cuales Heidegger había ganado terreno, la mayoría de las veces de un modo extremadamente solitario. Ella ha intentado reconciliar con el espíritu de la época la radicalidad, con la cual Heidegger reflexiona sobre palabras fundamentales como ser, verdad, tiempo o lenguaje. Con razón ella fue elogiada según eso con una acertada fórmula, como una urbanización de la provincia heideggeriana.

Ahora bien, justamente eso, que es para el espíritu civilizado de la época motivo de elogio, representa para la hermética filosófica un peligro y es motivo de inquietud. Pues justamente la hermética filosófica quiere advertir ante la urbanización, ante sus excesos y sus consecuencias perversas. No se duda, por cierto, de la legitimidad y de la fertilidad de la recepción hermenéutica de Heidegger. Pero la hermética filosófica insiste, por su lado, también con razón, sobre el hecho de que la radicalidad del pensar y de la crítica de la época, que llevó adelante Heidegger, resulta achatada en la recepción hermenéutica y no puede mantenerse como tal. Y así la hermética da preferencia al alma fenomenológica sobre la hermenéutica, aún cuando ambas en distintos planos residan en el corazón del pensar de Heidegger. La hermética quiere salvar, contra concesiones hermenéuticas al espíritu de la época, el convencimiento, que vive en la fenomenología y en Heidegger, de que la verdad no es producto [p. 268] de los esfuerzos acumulados por reconocer e interpretar, no es un constructo de un consenso, que se logra de modo hermenéutico-prudencial o discursivamente, sino que está antes de cualquier reconocer e interpretar, discutir y consensuar y solo puede ser puesto a disposición en un ver intuitivo y original. Solo sobre la base de ese acceso original dejan entonces ponerse en marcha también los procesos del comprender, de la interpretación, de la mediación, del entendimiento y de la construcción de consenso; y así toda hermenéutica tiene raíces, de modo inconsciente, en una hermética, que aquélla presupone y que yace en lo profundo. ${ }^{6}$ Todos esos procesos son, por fin, un mero acercamiento ciego al acontecer de la verdad, al cual ellos son solo capaces de perpetrarle un pequeño robo. Y solo el acceso a un ver original puede abrir a conocimientos más profundos en el acontecer de la verdad, que espera dar con ellos.

Seguramente, en el marco de una confrontación semejante, la hermética debe hacerse de una idea de la hermenéutica filosófica, en la cual seguro no se la vuelva a encontrar. Por ejemplo, así se la introduce: "La historia occidental está desde el comienzo bajo el dominio de un pensamiento determinado de luz y de ser, por el cual no ha llegado a la idea de que solo podría ser el pensar de una luz muy determinada y delimitada, y de un ser determinado y delimitado (...) En un mundo fundado en la comprensión, todo lo que es resulta interpretado

6 Sobre esto véase U. Eco, I limiti dell' interpretazione, Mailand 1990 (edición en alemán, München/ Wien 1992). 
como ser-conocido. Es manifestación según su propia naturaleza y se ofrece en el ámbito de un ser general, el cual es un ámbito de desocultamiento. Desocultamiento es el sentido del ser, y el conocimiento humano cumple este sentido en una proporción por cierto finita y limitada, pero legitimado y requerido en un sentido supremo. Esta legitimación se capta bajo el título verdad (...). Si a toda forma de conocer y de hacer patente se llama comprender, y a la ciencia del comprender se la llama hermenéutica, entonces a partir del contra-pensamiento [de la hermética] resulta de ese modo necesario preservar el ocultamiento, que en algún sentido es también 'doctrina', aunque ella tenga justamente por tema aquello, que está más allá de toda doctrina” (16). Pero ¿no era deseo de la hermenéutica despertar la conciencia [p. 269] de que todo texto presupone un contexto dador de sentido, que toda interpretación presupone un prejuicio que la constituya, que todo comprender presupone un horizonte, que todo bosquejo una perspectiva, en consecuencia toda espontaneidad presupone una pasividad como constitutiva? Pero más que de hermenéutica en su realidad, Rombach se ocupa de elucidar, de un modo especulativamente relevante, las relaciones de tensión entre hermética y hermenéutica. Pero démosle nuevamente la palabra a Rombach: "La hermética es la 'doctrina' de lo cerrado, tal como la hermenéutica es la doctrina de lo abierto y del desocultamiento. Esta contradicción se muestra de modo muy curioso. Dos disciplinas parientes por su origen se representan como oposición completa, cuando trazan sus respectivos objetivos. Esta curiosa relación se repite en las figuras de los dioses, aquí Hermes el dios de lo cerrado y el medio hermano y amigo Apolo, que es el más abierto y espléndido de los dioses griegos." (17) Incluso más pronunciadamente lo dice en otro pasaje: "En la medida en que el conocer hermético, que hace salir realidad recién a partir de sí, es posible solo sobre la base de que se quiebre un 'comprender' ya siempre dado, se debe pensar la hermética como una 'antihermeneútica'. 'Comprender' resulta para ella demasiado poco. Comprender queda afuera, apenas vuelve siempre sobre sí mismo, permanece en principio detenido. Estando así detenido y entendiendo el asunto hacia ese punto, donde está parado, pisa mal el asunto (ver-steht sie die Sache), lo destruye (steht sie zugrunde). En esa destrucción se apodera del asunto, lo vuelve disponible y se puede hablar al respecto. ${ }^{\mathrm{X}}$ Esta 'efectividad' parece justificar el pensar que comprende. En verdad, él es estéril, porque deja al hombre de lado, no lo mete dentro del movimiento del asunto, y no lleva al asunto a sobrepasar su propio nivel. La hermética capta sin 'método' pero todavía es, a decir verdad, el único 'camino' (méthodos), esto es, el camino que conduce al asunto, que el asunto mismo anda. Sólo aquel que pueda abandonar todos sus prejuicios logra ese auténtico movimiento, que es el sentido propio de la realidad. El ser humano puede estar realmente sin todos sus prejuicios, ciertamente solo en el feliz instante de un hallazgo hermético, en el cual quiebra todo aquello, que el hombre tenía a disposición hasta ese momento en los horizontes del reconocer y del obrar. La 
hermética es por eso también esencialmente carente de horizonte, y en absoluto se entiende como 'fusión de horizontes', tal como se acepta de la hermenéutica como fin de todo comprender y reconocer." (173)

[p. 270] Es especialmente acertado en la crítica epocal ese intento de esclarecer, en la polémica con la hermenéutica, el hecho de que tomar posesión de la herencia de Heidegger puede tener éxito no en las formas urbanas de trato y en las ceremonias, sino solo en un avance radical. Una urbanización de Heidegger debe hacer, pues, demasiadas concesiones al espíritu de la época para estar dispuesto a ofrecer ayuda para salir del estado patológico, en el cual ella se encuentra. Tan hábil, inteligente o fructífero que quiera ser siempre, compromiso y adaptación obligan, sin embargo, a hacer un tenue esbozo del problema, a suavizar la crítica heideggeriana relativa a los fundamentos del siglo, a dejar por ende los puestos más avanzados. Con la nivelación prosaica del contraste, a lo sumo, la hermenéutica lleva a cabo una corrección de la modernidad, pero ninguna puesta en discusión radical, tal como ésta se espera de la hermética a través de la dramatización de las tensiones.

En una coyuntura espiritual, en la cual palabras como crisis de valores y de sentido, desencantamiento del mundo, nihilismo, pensamiento negativo parecen siempre ocasionar cargas más pesadas y señalar inequívocamente un déficit fundamental, se atribuye a la hermética filosófica al menos el hecho de que ella busca ir al encuentro de ese déficit de modo radical. La hermética filosófica responde por eso también a una necesidad fundamental del siglo, por cierto sale al encuentro de esa necesidad de un modo inadecuado para la época, en el convencimiento de que a la época sólo puede serle de ayuda ir contra la época. Y así ella representa para el espíritu del tiempo una provocación en varios sentidos: contra posiciones de pensamiento resignadas respecto del acontecer de la verdad y de la realidad, ella se instala y se apoya en el todo y en lo absoluto, en la radicalidad y el fundamento; contra la absolutización de que el pensar esté ligado al lenguaje y al discurso, ella pretende restituir a éste la fuerza incólume de un ver originario; contra el trato seguro con el espíritu de la comunidad, contra el discutir, el acordar, el reunirse, ella aboga por el bello riesgo del andar audaz del filósofo, solitario y en la delantera.

¿Aceptará el siglo esta provocación?

$$
* * *
$$

\section{[p. 271] Querido Señor Volpi,}

me he traído su artículo acá arriba, a la cabaña, para leerlo con tranquilidad y para reflexionar, dado que me importa su opinión. Ahora estoy sentado en la sala y observo el remolino de nieve afuera, y ensayo una humilde respuesta, 
cuyo estado y forma, a causa de las circunstancias, le ruego sepa disculpar.

En primer lugar, le agradezco mucho el tiempo que Usted le ha dedicado a mis textos, y el gran esmero que ha tenido. Usted ha producido un trabajo excelente, y esto puede decirse muy rara vez de reseñas. Especialmente le agradezco la buena disposición que Usted ha tenido con mi trabajo, y que tampoco se haya dejado seducir por el elogio infundado. Usted ha tratado mis textos de modo crítico y también amistoso, los dejó hablar a ellos mismos, sin perder el control. Y tampoco ha evitado la difícil tarea de exponer la intención del autor, lo cual necesitaba de muchas páginas, al lograr resumirla en pocas páginas. ¡Le agradezco todo esto!

Dado que Usted me exige que yo mismo vuelva a tomar posición respecto de su toma de posición, quiero intentar tocar algunos puntos que me llamaron la atención.

En primer lugar está la cuestión de la posición de la hermética con respecto a mis otros trabajos. A decir verdad, ella no representa un "desarrollo" o "continuación" de mi punto de partida, sino que se trata de una de las cuatro "filosofías", que en cada caso yo he iniciado: ontología estructural, filosofía de la imagen (Bildphilosophie) (esto es, historia fundamental), fenomenología estructural y hermética. Ontología estructural y fenomenología forman un conjunto entre sí, pero siguen métodos diferentes. La filosofía de la imagen pertenece, en cambio, a otra tradición muy diferente y se apoya en otras formas de proceder. Nicolás de Cusa pertenece a la ontología estructural, Hölderlin a la filosofía de la imagen. Ambas no tienen nada en común, de lo cual resulta que ontología estructural y filosofía de la imagen son planteos distintos. La hermética se apoya además en otra tradición y proviene también de un interés completamente distinto: esa vez quise ver muy de cerca lo que era un "dios" (griego) y me atrapó casualmente Hermes. Allí se me abrió un paisaje completamente nuevo y caí de asombro en [p. 272] asombro, de un descubrimiento en otro. Pocas veces recibí tantos regalos como de la hermética. Naturalmente las cuatro "filosofías" forman un conjunto y se complementan también, pero ellas no se superponen; ninguna es el desarrollo posterior de la otra; cada una permanece completa y posee su propio territorio. En ese sentido la hermética puede ser tratada por sí sola y no necesita de otros textos para dilucidársela.

Me asombra un poco que Usted haya apenas tocado el punto crucial de la hermética; estoy pensando en la concepción, que cambia completamente la realidad respecto de toda la tradición filosófica. No considero las cosas como "entes" y los estados de cosas como "hechos" o como "lo que es el caso", sino como algo que podría llamarse, antes bien, "puntos cruciales" ("springende Punkte"), como indeterminaciones sumamente explosivas, cuyo potencial de realización para nada consiste en "posibilidad" en ellas. Asimismo, las "posibilidades" del ser humano ya no se encuentran en él, de modo tal que debiera 
esperarse solo al proceso de desenvolvimiento (desarrollo, despliegue, evolución, realización), sino que el ser humano es capaz de producciones imprevistas e "impensadas previamente" (Schelling) tanto como también las cosas son capaces de avances emergentes ${ }^{\mathrm{xi}}$ demasiado "imprevisibles", no "posibles". En todo se esconde algo que no se puede decir -esto es el descubrimiento ontológico y la declaración de la hermética-. Expresado de modo más técnico: cualquier asunto puede ser transportado a otra ontología y gana entonces otro nexo de posibilidad-realidad. Solo es posible pasar de la realidad a la posibilidad -y viceversa- en una determinada dimensión ontológica; si hay para la cosas (y para el hombre) un "salto" ontológico, entonces también hay el llamado (hermético) de fuerzas impensadas, entonces aquello, que antes se llamaba hechicería, lo cual es, sin embargo, una posibilidad completamente "real" recién cuando la "realidad" brota ontológicamente pluridimensional. En ese caso ella gana la posibilidad fundamental de la "elevación de sí" y "trascendencia de sí". Pero esto se ve recién cuando se "cambia totalmente el pensamiento sobre la realidad" (umdenken über die Wirklichkeit). Usted se topa un poco con esto en el subtítulo, pero éste expresa la intención del libro de modo exacto y correcto. Aquí no se exige más "constatar lo que es", o "decir" o "reconocer" o "tratarlo", sino concederle las propias posibilidades de creación: el tránsito de la hierba al cereal, [p. 273] desde el caballo ancestral del tamaño de un perro al animal de montura, de la bóveda hecha de barro del arte románico (a través del "arte de cortar piedras") a la magnífica cúpula del gótico, etc. De ese modo, y no a través de un mero despliegue, el hombre ha recorrido el camino desde el reptil al ser humano. Y esto contiene más hechizo que cualquier otra hechicería imaginada. El secreto, a través del cual son resueltos los saltos ontológicos es, tal como lo he descubierto y señalado en la hermética, la concreatividad. Y esto no quiere decir "ir de la mano", tal como nosotros lo pensamos quizá en el estilo de pensar desarrollado hasta el momento, sino un movimiento común (mejor: indiviso), en el cual ser humano y cosa forman una cosa, esto es, una estructura, y así a partir del mismo movimiento resultan ellos nuevos. No hay ni "lo que es", ni el "ser humano"; ambos son productos de desintegración, que se forman recién como entidades independientes, cuando el movimiento (génesis) ha terminado. A nuestros ojos parece diferente, pero sólo para el ojo "tonto", que ha aprendido a ver mal a través de un pensar equivocado. Un ojo agudo ve completamente claro que, por ejemplo, en el caso de aquella mujer negra que lleva su pesada carga sobre la cabeza manteniendo el equilibrio, ser humano y carga se pertenecen, recién juntos hacen al "humano" (y, es decir, bello) carácter del andar. Un mono no "carga" nada, aún cuando se le ate una carga. Así "cargar" (entre muchas otras cosas) es el nacimiento del hombre, al mismo tiempo también el nacimiento de cosas valiosas, porque se carga solo lo que es "valioso". Ser humano y mundo de valor van uno con otro y emergen 
uno a partir del otro, y no existe primero el hombre que luego cree (schafft) un mundo de cosas valiosas. No hay "ser humano", eso es un engaño imbécil, y no hay "cosas", ellos dejan salir de sí mundos que han transformado la faz de la tierra. A esto llamo verdaderamente un cambio total en el pensar (umdenken), si debo concebir y pensar la realidad sin "ser humano" y "cosas".

Ahora bien, sostengo que los hombres ya están por sí mismos en camino de semejante nueva comprensión de la realidad. Es decir, están unidos todos los movimientos “alternativos", que todavía no han capturado su oculta conexión. Todos toman parte de algún nodo de la experiencia fundamental hermética, y toman de ella respectivamente desde distintos lados. Así ya los hippies, los partidarios de las comunas, el movimiento pacifista, el movimiento ecológico, los partidarios de la democracia directa, también los excluidos por cierto, los adictos a las drogas y las colectividades de pacientes. Por eso: mundo y contra-mundo. Como contra-mundo se ha [p.274] ya entendido el "movimiento alemán", que en 1800 dio el primer paso en dirección a transformar la comprensión de la realidad. Todo esto fue visto en conjunto por mí por vez primera y, tal como yo creo, encierra la actualidad del libro. Pues bien, apenas si alguien lo ha notado, aunque sea emocionante -o me equivoco acaso-. En muchas partes se clama por una "filosofía" de los alternativos o también de los verdes; bueno, aquí está ella. Con esto, los verdes y los alternativos deberían, no obstante, desistir de alguna travesura, y esto los deja sordos.

Desde aquí se comprende mejor también cómo me manejo con la pregunta por la crítica epocal. (Usted introduce su reseña con esta pregunta.) Yo no hago ninguna crítica epocal, sino que describo la crítica, que la época se propone sobre sí misma. El "cambio radical (Umbruch)" acontece, y yo solo necesito tocar su propia melodía para sacarlo a bailar. Esta es la "gaya ciencia", tal como la entiendo. A partir de esto se entiende también mi optimismo, el cual aparece en su reseña algo carente de razón. Como la hermética es ella misma el carácter de la realidad, no se necesita que se la "realice". Si el camino desde la rana al ser humano fue recorrido de ese modo, el próximo paso bien podrá ser logrado. Por lo demás, veo tantos indicios (véase más arriba), de modo tal que estoy libre de cualquier preocupación.

Una palabra más sobre la "posmodernidad". Dado que el cambio de pensar (umdenken) sobre la realidad, que pienso y describo, va en contra de los fundamentos de la filosofía greco-occidental, aquel alcanza la historia europea en conjunto. Todas sus épocas juntas son el Eón ${ }^{x i i}$, que cierra y debe ser reemplazado por otro nuevo. El nuevo tiempo caracterizado por la experiencia fundamental hermética de ningún modo se opone solo a la modernidad. Si se ve el cambio radical (Umbruch) solo como algo semejante hacia la posmodernidad, entonces se toca un período muy breve, demasiado breve. Esta idea es para mí valiosa, y podría tener un significado en la disputa contemporánea 
acerca del cambio radical. Por eso aquí hablo de esto, a pesar de que Usted no haya rozado esta cuestión.

Algo más. Siempre me ha asombrado cuántos "descubrimientos" pueden plantearse, sin que algo se mueva en el mundo de los lectores. Así Leben des Geistes $^{x i i i}$ está atestado de descubrimientos, la mayoría de los cuales deberían mantenerse firmes. Pero nadie los ha agradecido. De modo semejante creo que la hermética ha sido adoquinada con esos descubrimientos, [p. 275] si pienso solo por caso en las distintas "interpretaciones": Chagall, Max Ernst, Bosch, Durero, etc. ¿Pues dónde han sido Techos rojos o El nadador ahogado o Regreso al hogar materia de tanta instrucción? Ni siquiera Heidegger, que es por lo demás un maestro de las interpretaciones herméticas, ha descubierto el secreto de Regreso al hogar. Me gustaría discutir al respecto alguna vez con alguien entendido.

Ahora una palabra más sobre la relación entre hermética y hermenéutica. He mantenido correspondencia también al respecto con Gadamer, quien parece "aceptar el desafío" y pretende haber escrito algo sobre el tema. Sin embargo, no me puedo imaginar esto realmente, porque él no tiene ojo para eso, se supone que no ha visto nunca un Hérmaion ${ }^{x i v}$, él siempre ha "comprendido" apenas. Esto se indicaba también de modo concreto en la diferencia de interpretación de un poema de Hölderlin (Heidelberg), donde él no llegó a captar el "hechizo" allí descripto. Ahora bien, no quiero "hablar mal" de Gadamer, él tiene sus méritos, de los cuales Usted habla por cierto, pero el hecho de que su obra principal se le fuera de las manos de un modo inadvertido, es sin embargo algo increíble. Comienza ciertamente como una hermética -y el título "Verdad y Método" mienta, sin embargo, una oposición-: él busca la verdad que puede ser alcanzada sin método, y esto es nada menos que la hermética. Pero luego resulta un método de ella y en realidad el libro sí que es leído ciertamente como un libro del método de la hermenéutica, en lo cual también se convierte solo después de las primeras páginas. Pero es, sin duda, un escándalo que los lectores no se hayan dado cuenta de esa inversión.

La oposición de hermética y hermenéutica no se capta a partir de la "urbanidad", o aquélla se representa en relación a ésta justo al revés. Hermética es la captación de mundos, hermenéutica es el trabajo de interpretación dentro de un mundo, en el cual no se ve el carácter del mundo (es decir, el hecho de que ese único mundo esté cerrado y separado de otros posibles). Se piensa que se está en el mundo -y que hay naturalmente sólo un único mundo, justamente aquel, en el cual uno mismo se encuentra-. Esto es naturalmente un error, se absolutiza este único mundo, en el cual uno casualmente se encuentra, y todos los otros, que se encuentran también allí, acuerdan con eso. Esto parece urbano, pero es totalmente provinciano. Realmente urbano es sólo el hermético, que ve más allá de los límites de su mundo y ha querido ver otros mundos como otros 
mundos y está también dispuesto a ello. Sólo quien [p. 276] vuelve de regreso de semejantes "viajes" (Hermes como Dios del viaje) -de viajes a otros mundos-, es un "hombre de mundo" Los que se quedan en casa son "torpes" y siguen así.

El pluralismo y las multiveracidad de los mundos no se manifiestan naturalmente como multiplicidad en simultaneidad. Mundos hay de vez en cuando y solo de modo único. Semejante relación, que no es monista ni pluralista, de aquí tampoco "posmoderna", no se ha pensado nunca en la filosofía europea. En la hermética se piensa, se describe, se ejecuta con alegre naturalidad.

Esta es, en suma, la filosofía que se hace. Ella no habla, ella es. Por eso ella parece callar para otras filosofías, o solo apenas ofrece "alusiones", "indicaciones", "ejemplos" ilustrativos. En verdad, ella muestra, y por cierto completamente y sin ocultar nada. Sólo un hermético puede realmente mostrar, y el hecho de que usemos la metáfora "mostrar" también para procedimientos hermenéuticos como, por ejemplo, demostraciones, "muestra" de vuelta que la hermenéutica se funda en una hermética (sin que aquella lo sepa). Al respecto habría mucho que decir, sin que ese decir pudiera "mostrar" algo, si el oyente no ha "visto" ya de antemano.

Una vez más le agradezco, cordiales saludos,

Suyo

Heinrich Rombach

Notas de La traducción (Por Graciela M. Chichi)

[i] Hay edición española: H. RomBAch, El presente de la Filosofía. Los problemas fundamentales de lafilosofía occidental y el estado actual de las cuestiones filosóficas, Barcelona: Herder, 2007, 197 pp.

[ii] La reciente edición de estudio tiene nuevo subtítulo: Substanz, System, Struktur: Die Hauptepochen der europäischen Geistesgeschichte, 2 Bände, Freiburg im Briesgau: Verlag Karl Alber, 2010.

[iii] Dejo transliterado el neologismo konkreativ, de uso técnico en Rombach y que Volpi refiere.

[iv] Asimismo translitero el neologismo Idemität.

[v] El verbo umdenken significa un cambio en los fundamentos del pensar, poner en juego nuevas reflexiones. Véase Duden. Deutsches Universalwörterbuch, Bearbeitet von prof. Dr. G. DrosDowski u.a., 2. Auflage, Manheim u.a.: Dudenverlag, 1989, p. 1588, columna a.

[vi] De aquí en adelante traduzco las citas del $W u G W$ de Rombach, al hilo de la selección de Volpi. Entre paréntesis figuran las páginas de esa obra. [vii] La edición española es: H. RomBach, El hombre humanizado. Antro- 
pología estructural, Barcelona: Herder Editorial, 2004, 480 pp.

[viii] En la cita se habla de Aufklärung, que como otras veces traduje 'ilustración'.

[ix] Mi versión del término 'Ursprung' pretende retener lo conceptual de lo mucho y rico que Rombach deja sentado con su noción propia y bien delimitada respecto de los sentidos acuñados en la historia de la filosofìa. Por lo pronto, el Ursprung acontece en la experiencia hermética, que como tal sobrepasa los límites del mundo. En esa experiencia se pone en juego algo nuevo, que anuncia otro mundo en lo profundo. Nuevo es, pues, aquello que no deja entenderse ni integrarse a los horizontes de expectativas (previos), a conexiones cotidianas de mundo conocido. Cuando lo nuevo emerge o surge -diríamos- ello mismo abre las condiciones de su experiencia. En sus palabras: Das Neue, das so aufspringt, dass es auch noch die Bedingungen seiner Erfahrung eröffnet, wird in unserer Sprache Ursprung gennant. (WuGW, 1983, p. 108b). "Es el comienzo (Beginn) de eso nuevo, en sentido propio: el comienzo del camino (Gang) de algo." Y entonces Rombach precisa: "Ursprung no es fundamento (Grund). Fundamento es un modo de apertura en una conexión de apertura ya existente. Ursprung es la inauguración/apertura (Eröffnung) de una nueva conexión de apertura en un lugar concreto y a partir de su propia posibilidad. Ursprung es el comienzo a partir del "camino" de las cosas, no algo "desde el comienzo" (von Anfang an). (...) El fundamento puede tener lugar recién dentro de esa nueva apertura; de aquí que los fundamentos ya sean cosas muy deducidas." (ibid.). Entre una idea y otra Rombach afirma que entender de modo originario, original (ursprünglich) un drama, en tanto acontecimiento hermético, no consiste en contarlo a partir de que comienza la obra, sino, antes bien, desde el punto en el que se pone en marcha el cambio respecto de otro/s punto/s. En todo caso, a partir de este camino retornamos de nuevo al principio de la obra. "Drama es un acontecer en el que todo se da vuelta (alles wendet), viraje (Umwendung) peripecia." (ibid.). Véase, ROMBACH, op.cit. sección V .1 titulada Einbruch und Ursprung, pp. 108-110. La última cita saca provecho de la famosa noción de La Poética (cap. 11) de Aristóteles.

[x] Mi versión del pasaje intenta volcar juegos de palabras, a partir de los términos 'verstehen' y 'Standpunkt', con los cuales Rombach hace valer la inoperancia del comprender (verstehen) respecto del asunto del pensar.

[xi] En el texto figura el plural de 'Hervorgang'.

[xii] Rombach se refiere al Aiẃn griego, que en español se dice "eón", véase la $D R A E$ bajo esa entrada.

[xiii] Se refiere a su libro.

[xiv] En griego, el sustantivo neutro ‘hérmaion, -ou' significa hallazgo afortunado, así lo atestiguan pasajes de Sófocles hasta de Platón, entre otros. Véase A. BAILly, Dictionnaire grec-français, Paris: Librairie Hachette, 1950, 
s.v. p. 806 b. Por su lado, sustantivos como ‘hérma, -atos' o 'érmai' nombran las piedras o mojones, con las que se señalaban las bifurcaciones de los caminos, o los límites de las propiedades. Véanse ibid. p. $806^{\mathrm{a}}$ y s.v. 'Epuńs, -oû, p. $807^{\mathrm{a}}$. Se supone que el nombre de 'Hermes' se remonta a la referencia de los mojones, por lo cual sería la divinidad de los límites de los mundos, que incluye el subterráneo.

AGRADECIMIENTOS: En primer lugar, agradezco a Norbert A. Balk ( $M$. A.), quien puso en mis manos el material, me ayudó a corregir una de las últimas versiones de mi traducción y tuvo la generosidad de entrar en contacto con los responsables de la publicación original del artículo. Agradezco al profesor Georg Stenger, en nombre de los editores académicos, y al Sr. Lukas Trabert de la Verlag Karl Alber, por haber autorizado la traducción del material por ellos publicado y cedido en forma gratuita los derechos de una eventual edición de una versión en castellano, cuya propuesta sometí a referato en la Revista Contrastes. Y a Romina Bedogni Drago, por haber mejorado el inglés de mi resumen. Finalmente, a la memoria del profesor Franco Volpi -quien supo de nuestra intención de dar a conocer su trabajo en nuestra lengua- a pesar de que no llegásemos a entregarle la traducción terminada; con sincero reconocimiento.

Graciela Marta Chichi es profesora Titular ordinaria de la Universidad Nacional de La Plata,

CONICET, Argentina.

Publicaciones recientes:

- «Acerca de las presentaciones de los textos de La Retórica y La Poética de Aristóteles», Opúsculos Filosóficos (publicación del Centro de Filosofía Clásica de la Facultad de Filosofía y Letras de la Universidad Nacional de Cuyo) Mendoza, Argentina, Año VI, N 18, (2013), pp. 13-54. ISSN 1852-0596.

-«El tratamiento aristotélico de la diabolé en La Retórica entre la primera reflexión retórica», Kléos. Revista de Filosofia Antiga (publicación anual del Programa de Estudos em Filosofia Antiga del Instituto de Filosofía y Ciencias Sociales de la Universidade Federal do Rio de Janeiro), Brasil. 15, año 2011 (2014), pp. 27-51. URL http://www.pragma.ifcs.ufrj.br/ kleos15 ISSN: 2236-0077.

Correo electrónico: gchichi@isis.unlp.edu.ar 